\title{
Optimal unbiased estimation of some population central moments
}

\author{
Mariano Ruiz Espejo • Miguel Delgado Pineda • \\ Saralees Nadarajah
}

Received: 20 December 2012 / Accepted: 11 February 2013 / Published online: 28 May 2013

(C) Sapienza Università di Roma 2013

\begin{abstract}
In this paper, we have treated the problem of estimating some population central moments under distribution-free setting. Uniformly minimum variance unbiased estimators for some population central moments have been derived. Some examples of unbiased estimators of central moments have been given under various sampling designs such as simple random sampling with replacement ( $s r s r$ ) or without replacement ( $s r s)$, probability proportional to size with replacement ( $p p s r$ ) and probability graduated variable proportional to size without replacement ( $p g v p s$ ). An optimal unbiased estimator of the third population central moment is proposed and extended to some real situations. Some optimal unbiased estimators of the fourth population central moment are given. Several optimal unbiased estimators of the variance of the "sample quasivariance estimator" are identified. Finally, computer programs in $\mathrm{R}$ implementing all of the estimators are given.
\end{abstract}

Keywords Distribution free setting · Optimal unbiased estimators · Population central moments $\cdot R \cdot$ Stratified sampling

\section{Introduction}

Suppose that we wish to study the distribution of a random variable. A good knowledge of the distribution can be obtained by knowing its first two moments, i.e., the mean and variance. If we have a random sample from the variable then the unbiased estimators for the mean and variance are well known and can be found in most statistics text books.

However, there are many situations that require moments of higher orders and not just the first two. For example, higher order moments are required in: signal classification [53], testing homogeneity among clustered data [25], estimating equations with nuisance parameters [64],

M. Ruiz Espejo · M. Delgado Pineda

Facultad de Ciencias, UNED, 28040 Madrid, Spain

S. Nadarajah $(\varangle)$

University of Manchester, Manchester M13 9PL, UK

e-mail: Saralees.Nadarajah@manchester.ac.uk 
generalized calibration approach for estimating variance [52], mismeasured regressor errorsin-variables models [12], empirical analysis of earnings and employment risk [15], Monte Carlo algorithms for semiparametric linear mixed models [24], modeling of the distribution of raindrop diameters [6], tests of multinormality based on location vectors and scatter matrices [27], non-data-aided signal to noise ratio estimation [30], structural equation modeling [48], parameter estimation for INAR processes [50], portfolio selection [21], analysis of student satisfaction towards university courses [35], linear panel data models with potential existence of time effects [62], estimation for linear mixed models [63], and estimation in linear mixed models for longitudinal data under linear restricted conditions [29].

However, there has not been much research to find unbiased estimators for moments or central moments. The work that we are aware of are Dwyer [11], Halmos [18], de Lucia [8], Gupta [16,17], Mikhail and Malik [34], Herzel [23], Heffernan [22], Rose and Smith [44], Singh et al. [51] and Kadilar et al. [26]. Most of these papers are old. The recent ones either do not provide unbiased estimators or are based on complicated concepts like $h$-statistics and $U$-statistics, which can be computationally expensive.

For instance, Herzel [23] gives an unbiased estimator for a general symmetric function under simple random sampling. Heffernan [22] gives a general estimator for the $r$ th central moment. In the words of Heffernan [22], the estimator involves a sum that "extends over all $n ! /(n-r)$ ! permutations $\left(i_{1}, \ldots, i_{r}\right)$ of $r$ distinct integers chosen from $\{1, \ldots, n\}$ ". This can be computationally expensive if $n$ is large.

In practice, general formulas are not very useful. Most practitioners may not have the mathematical depth to understand or use the general estimators given by Herzel [23], Heffernan [22] and others. We are not sure if general estimators can be implemented in some software. In any case, Herzel [23] and Heffernan [22] do not appear to have implemented their estimators in any software. Such implementations could have given their estimators some practical appeal.

Most situations in practice only require estimators for the first four central moments. So, it is much more useful to have explicit formulas for unbiased estimators for the first four central moments. Unbiased estimators for the second central moment are given by Theorem 2.1. The estimators given by this theorem apply for $p p s r$ and $p g v p s$ designs incorporating stratification, but the estimators can be extended easily for other designs too. Unbiased estimators for the third central moment are given by Theorems 3.1 and 3.2. The estimators given by these theorems apply for srsr designs incorporating stratification, but the estimators can be extended easily for other designs too. Unbiased estimators for the fourth central moment are given by Theorem 4.1. The estimators given by this theorem apply for srsr designs, but the estimators can be extended easily for other designs too.

As seen later, apart from being more useful, the estimators given by Theorems 2.1, 3.1, 3.2 and 4.1 are much simpler than those given by Herzel [23] and Heffernan [22]. Hence, they can be readily used by practitioners of sampling designs. To aid the practical use, we have given simple functions in R [40] for computing the unbiased estimators given by Theorems 2.1, 3.1, 3.2 and 4.1, see Sect. 5. We have chosen $R$ to write these functions because, unlike other statistical software, it is freely downloadable from the Internet (http://www.r-project.org). The functions could have wide applicability because they are simple and easy to implement on any platform and full control is given to the user as far as accuracy and stability of the results.

There are several contributed packages in $\mathrm{R}$ for sampling designs. The ones we are aware of are: SamplingStrata [3]; spsurvey [28]; survey [31]; SunterSampling [2]; samplingVarEst [13]; SDaA [60]; lavaan.survey [37]; odprism [59]; osDesign [19]; stratification [1]; StatMatch [10]; RDS [36]; endorse [49]; list [5]; SvyNom [14]; samplingbook [32]; 
TeachingSampling [43]; sampling [57]; reweight [7]. None of these packages appear to give functions for unbiased estimation of central moments. The functions given in Sect. 5 appear to be the first of their kind.

Another novelty of our work relates to the pgvps sampling design introduced by Sánchezsps Crespo [47]. The properties of this sampling design have not been followed up in the literature. Sánchezsps Crespo [47] illustrated importance of the pgvps sampling design to areas such as "printing with or without discount in the ballot box". We derive here for the first time unbiased estimators of central moments under pgvps. if

An estimator $\widehat{\theta}=\widehat{\theta}\left(x_{1}, x_{2}, \ldots, x_{n}\right)$ is unbiased for the population parameter $\theta$ if and only

$$
E_{F}(\widehat{\theta} ; \theta)=\theta
$$

for all distribution functions $F$ for which the expectation, $E_{F}(\widehat{\theta} ; \theta)$, exists. Moreover, the unbiased estimator $\widehat{\theta}=\widehat{\theta}\left(x_{1}, x_{2}, \ldots, x_{n}\right)$ for $\theta$ is of minimum variance uniformly if and only if

$$
V_{F}(\widehat{\theta} ; \theta) \leq V_{F}(e ; \theta)
$$

for all unbiased estimators $e=e\left(x_{1}, x_{2}, \ldots, x_{n}\right)$ and for all distribution functions $F$ for which $V_{F}(e ; \theta)$, the variance of $e$ for estimating $\theta$, exists. The terms "for all distribution function $F$ " refer to "distribution-free setting", i.e., the sample $\left(x_{1}, x_{2}, \ldots, x_{n}\right)$ is an $n$-dimensional real valued vector from $\left(X_{1}, X_{2}, \ldots, X_{n}\right)$, an $n$-dimensional random variable, with $X_{i}$ and $X_{j}$ (for $i \neq j$ ) independent and identically distributed random variables from a common and unknown (distribution-free) function $F$.

In this paper, we provide a general method for unbiased estimation of population central moments. The suggested method provides an unbiased and optimal estimator of the 'population variance' (population central moment of order two) for a distribution-free setting.

For the third population central moment, there is an unbiased estimator whose variance decreases asymptotically if the sample size increases to infinity (i.e., if $n \rightarrow \infty$ ). This estimator is also optimal under distribution-free unbiased setting (see Theorem 3.1). Optimal unbiased estimators of the fourth population central moment are also obtained under distribution-free setting (see Theorem 4.1).

Some results have been extended further to various sampling designs for finite populations.

When the complete sample is not yet available for obtaining 'optimal unbiased estimators of population central moments' (OUEPCM), we can estimate them unbiasedly (see Theorems 3.1 and 4.1) if we know the successive 'optimal unbiased estimators of the population noncentral moments' (OUEPNCM). So, when the complete sample data are not yet at hand, the population central moments describing dispersion, asymmetry and skewness can be estimated from the available OUEPNCM. The origin of this research was the study of the distribution of Spanish family incomes.

Bellhouse [4], Stafford and Bellhouse [54,55] and Rose and Smith [44] have developed computer algebra systems to compute expected values under any unistage sampling and their unbiased estimators. It is not clear whether their computer algebra systems can be used to compute unbiased estimators for moments and central moments of any order. Furthermore, their systems are implemented in Mathematica [61], a commercial package. Most practitioners of sampling design may not have or may not be familiar with Mathematica. They would prefer having an explicit mathematical formula that can be readily implemented in a pocket calculator or a non-commercial package like R, see Sect. 5. 


\section{Population variance}

Let $F$ be the distribution function of a random variable $X$. The population variance of $X$ is then defined by

$$
\sigma^{2}=\mu_{2}=\int_{-\infty}^{\infty}(x-\mu)^{2} d F(x)(<\infty)
$$

where

$$
\mu=\alpha_{1}=\int_{-\infty}^{\infty} x d F(x)
$$

is the population mean.

In general,

$$
\alpha_{k}=\int_{-\infty}^{\infty} x^{k} d F(x) \quad \text { and } \quad \mu_{k}=\int_{-\infty}^{\infty}(x-\mu)^{k} d F(x)
$$

where $k$ being a positive integer. Here and throughout, the integration is with respect to an underlying superpopulation model.

Lemma 2.1 If $\widehat{\alpha}_{k}$ and $\widehat{V}\left(\widehat{\alpha}_{k}\right)$ are, respectively, unbiased estimators of $\alpha_{k}$ and $V\left(\widehat{\alpha}_{k}\right)$, an unbiased estimator of the population variance $\mu_{2}$ is

$$
\widehat{\mu}_{2}=\widehat{\alpha}_{2}-\widehat{\alpha}_{1}^{2}+\widehat{V}\left(\widehat{\alpha}_{1}\right) .
$$

Proof As $E\left(\widehat{\alpha}_{2}\right)=\alpha_{2}, E\left(\widehat{\alpha}_{1}^{2}\right)=\alpha_{1}^{2}+V\left(\widehat{\alpha}_{1}\right)$ and $E\left\{\widehat{V}\left(\widehat{\alpha}_{1}\right)\right\}=V\left(\widehat{\alpha}_{1}\right)$ :

$$
E\left(\widehat{\mu}_{2}\right)=\alpha_{2}-\left\{\alpha_{1}^{2}+V\left(\widehat{\alpha}_{1}\right)\right\}+V\left(\widehat{\alpha}_{1}\right)=\alpha_{2}-\alpha_{1}^{2}=\mu_{2} .
$$

Hence, the proof.

Example 2.1 For a simple random sample of size $n$ drawn with replacement (srsr), we consider

$$
\widehat{\alpha}_{k}=\frac{1}{n} \sum_{i=1}^{n} x_{i}^{k},
$$

and

$$
\widehat{\mu}_{2}=\left(\widehat{\alpha}_{2}-\widehat{\alpha}_{1}^{2}\right)+\widehat{V}\left(\widehat{\alpha}_{1}\right)=\frac{n-1}{n} s^{2}+\frac{\widehat{\mu}_{2}}{n},
$$

where $\widehat{\mu}_{2}=s^{2}$ is the sample quasivariance. The estimator

$$
s^{2}=\frac{1}{n-1} \sum_{i=1}^{n}\left(x_{i}-\widehat{\alpha}_{1}\right)^{2}
$$

is also unbiased and optimal under a distribution-free setting, or it is an UMVU estimator of $\sigma^{2}$ (see [65, page 150]). We now consider other sampling designs. 
Example 2.2 Under probability proportional to size with replacement ( $p p s r$ ) design of a finite population (see [20]), we deduce:

$$
\widehat{\alpha}_{k}=\sum_{i=1}^{n} \frac{x_{i}^{k}}{n N P_{i}} \quad \text { and } \quad \widehat{V}\left(\widehat{\alpha}_{k}\right)=\frac{1}{n(n-1) N^{2}} \sum_{i=1}^{n}\left(\frac{x_{i}^{k}}{P_{i}}-N \widehat{\alpha}_{k}\right)^{2} \text {, }
$$

where $\sum_{i=1}^{N} P_{i}=1$, and $N(<\infty)$ is the finite population size.

The estimator of Lemma 2.1, $\widehat{\mu}_{2}=\widehat{\alpha}_{2}-\widehat{\alpha}_{1}^{2}+\widehat{V}\left(\widehat{\alpha}_{1}\right)$, generalizes the srsr design for $P_{i}=1 / N(i=1,2, \ldots, n)$; see Example 2.1.

Example 2.3 Under probability graduated variable proportional to size without replacement (pgvps) design (see [47]) of a finite population, we deduce:

$$
\widehat{\alpha}_{k}=\sum_{i=1}^{n} \frac{x_{i}^{k}}{n N P_{i}}
$$

where $P_{i}=M_{i} / M ; M_{i}$ and $M$ are positive natural numbers such that

$$
\sum_{i=1}^{N} P_{i}=1
$$

The unbiased variance estimator of $\widehat{\alpha}_{k}$ is given by

$$
\widehat{V}\left(\widehat{\alpha}_{k}\right)=\frac{M-n}{M} \frac{1}{n(n-1) N^{2}} \sum_{i=1}^{n}\left(\frac{x_{i}^{k}}{P_{i}}-N \widehat{\alpha}_{k}\right)^{2} .
$$

So, from Lemma 2.1, the respective unbiased estimator $\widehat{\mu}_{2}$ can be obtained. This result reduces to the case of simple random sampling without replacement (srs) design, for $P_{i}=1 / N$ and $M=N$.

Theorem 2.1 Under ppsr or pgvps designs of size $n_{h}$ independently in each stratum $h$, an unbiased estimator of $\mu_{2}$ is:

$$
\widehat{\mu}_{2}=\sum_{h=1}^{L} W_{h} \widehat{\alpha}_{2 h}-\left(\sum_{h=1}^{L} W_{h} \widehat{\alpha}_{1 h}\right)^{2}+\sum_{h=1}^{L} W_{h}^{2} \widehat{V}\left(\widehat{\alpha}_{1 h}\right),
$$

where $W_{h}=N_{h} / N$ is the relative size of stratum $h(h=1,2, \ldots, L)$, and $\widehat{\alpha}_{k h}$ and $\widehat{V}\left(\widehat{\alpha}_{1 h}\right)$ are unbiased estimators of the noncentral moment of order $k$ in the stratum $h$, and, respectively, for the variance $V\left(\widehat{\alpha}_{1 h}\right)$, for a common sampling design in each stratum $h$. 
The proof is immediate from Lemma 2.1.

Remark 2.1 For srs design in each stratum it is obvious from Theorem 2.1 that

$$
\begin{aligned}
\widehat{\mu}_{2} & =\sum_{h=1}^{L} \frac{W_{h}}{n_{h}} \sum_{i=1}^{n_{h}}\left(x_{h i}-\widehat{\alpha}_{1}\right)^{2}+\widehat{V}\left(\widehat{\alpha}_{1}\right) \\
& =\sum_{h=1}^{L} \frac{W_{h}}{n_{h}} \sum_{i=1}^{n_{h}} x_{h i}^{2}-\widehat{\alpha}_{1}^{2}+\widehat{V}\left(\widehat{\alpha}_{1}\right) .
\end{aligned}
$$

This result is due to Rao [42]. We denote $\widehat{\alpha}_{1}=\bar{x}_{s t}=\sum_{h=1}^{L} W_{h} \widehat{\alpha}_{1 h}, \widehat{\alpha}_{1 h}=\bar{x}_{h}$ being the sample mean in the stratum $h$. The Rao's estimator, $\widehat{\mu}_{2}$, is also unbiased for srsr or srs designs in each stratum, where the selections are independent in different strata.

Remark 2.2 For a proportional allocation $\left(n_{h}=n W_{h}, h=1,2, \ldots, L\right)$ with $s r s r$ or $s r s$ designs, we have the unbiased estimator

$$
\widehat{\mu}_{2}=\sigma_{s}^{2}+\widehat{V}\left(\bar{x}_{s t}\right),
$$

where

$$
\sigma_{s}^{2}=\frac{1}{n} \sum_{h=1}^{L} \sum_{i=1}^{n_{h}}\left(x_{h i}-\bar{x}_{s t}\right)^{2} \quad \text { and } \quad \bar{x}_{s t}=\frac{1}{n} \sum_{h=1}^{L} \sum_{i=1}^{n_{h}} x_{h i}
$$

are, respectively, the sample variance and the sample mean.

Remark 2.3 In case of estimating a 'population proportion' $P$ in stratified sampling, with an $s r s r$ or srs design independently in each stratum, the random variable $X$ takes only 'zero' or 'one'. We deduce the unbiased estimator

$$
\widehat{\mu}_{2}=\bar{x}_{s t}\left(1-\bar{x}_{s t}\right)+\widehat{V}\left(\bar{x}_{s t}\right) .
$$

\section{Population central moment of order three}

In this section, we consider estimation of $\mu_{3}$.

Lemma 3.1 If $\widehat{\omega}$ denotes an unbiased estimator of $\omega$ (see, for example, [45], an unbiased estimator of $\mu_{3}$ is

$$
\begin{aligned}
\widehat{\mu}_{3}= & \widehat{\alpha}_{3}-3 \widehat{\alpha}_{2} \widehat{\alpha}_{1}+3 \widehat{\operatorname{Cov}}\left(\widehat{\alpha}_{2}, \widehat{\alpha}_{1}\right)+2 \widehat{\alpha}_{1}^{3} \\
& -2 \widehat{\alpha}_{1} \widehat{V}\left(\widehat{\alpha}_{1}\right)-2 \widehat{\operatorname{Cov}}\left(\widehat{\alpha}_{1}^{2}, \widehat{\alpha}_{1}\right)+2 \widehat{\operatorname{Cov}}\left\{\widehat{\alpha}_{1}, \widehat{V}\left(\widehat{\alpha}_{1}\right)\right\} .
\end{aligned}
$$

Proof We have

$$
\begin{aligned}
E\left(\widehat{\alpha}_{3}\right) & =\alpha_{3}, \\
E\left(\widehat{\alpha}_{2} \widehat{\alpha}_{1}\right) & =\alpha_{2} \alpha_{1}+\operatorname{Cov}\left(\widehat{\alpha}_{2}, \widehat{\alpha}_{1}\right), \\
E\left(\widehat{\alpha}_{1}^{3}\right) & =E\left(\widehat{\alpha}_{1}^{2} \widehat{\alpha}_{1}\right) \\
& =E\left(\widehat{\alpha}_{1}^{2}\right) \alpha_{1}+\operatorname{Cov}\left(\widehat{\alpha}_{1}^{2}, \widehat{\alpha}_{1}\right) \\
& =\left\{\alpha_{1}^{2}+V\left(\widehat{\alpha}_{1}\right)\right\} \alpha_{1}+\operatorname{Cov}\left(\widehat{\alpha}_{1}^{2}, \widehat{\alpha}_{1}\right) \\
& =\alpha_{1}^{3}+\alpha_{1} V\left(\widehat{\alpha}_{1}\right)+\operatorname{Cov}\left(\widehat{\alpha}_{1}^{2}, \widehat{\alpha}_{1}\right),
\end{aligned}
$$


and

$$
E\left\{\widehat{\alpha}_{1} \widehat{V}\left(\widehat{\alpha}_{1}\right)\right\}=\alpha_{1} V\left(\widehat{\alpha}_{1}\right)+\operatorname{Cov}\left\{\widehat{\alpha}_{1}, \widehat{V}\left(\widehat{\alpha}_{1}\right)\right\}
$$

So,

$$
E\left(\widehat{\mu}_{3}\right)=\alpha_{3}-3 \alpha_{2} \alpha_{1}+2 \alpha_{1}^{3}=\mu_{3}
$$

Hence, the proof.

Theorem 3.1 For srsr designs of size $n$, if

$$
\widehat{\alpha}_{k}=\frac{1}{n} \sum_{i=1}^{n} x_{i}^{k}(k=1,2,3) \text { and }-\infty<\mu_{3}<\infty,
$$

then, the optimal unbiased estimator of $\mu_{3}$ under distribution-free setting is

$$
\widehat{\mu}_{3}=\frac{n^{2}}{n^{2}-3 n+2}\left(\widehat{\alpha}_{3}-3 \widehat{\alpha}_{2} \widehat{\alpha}_{1}+2 \widehat{\alpha}_{1}^{3}\right) \text {. }
$$

Proof We have:

$$
\widehat{\operatorname{Cov}}\left(\widehat{\alpha}_{2}, \widehat{\alpha}_{1}\right)=\frac{\left(\alpha_{3}-\widehat{\alpha_{2}} \alpha_{1}\right)}{n}=\frac{\widehat{\alpha}_{3}}{n}-\frac{\widehat{\alpha}_{2} \widehat{\alpha}_{1}-\widehat{\operatorname{Cov}}\left(\widehat{\alpha}_{2}, \widehat{\alpha}_{1}\right)}{n}
$$

which implies

$$
\widehat{\operatorname{Cov}}\left(\widehat{\alpha}_{2}, \widehat{\alpha}_{1}\right)=\frac{\widehat{\alpha}_{3}-\widehat{\alpha}_{2} \widehat{\alpha}_{1}}{n-1}
$$

Further,

$$
\begin{aligned}
\widehat{\operatorname{Cov}}\left(\widehat{\alpha}_{1}^{2}, \widehat{\alpha}_{1}\right) & =\widehat{\operatorname{Cov}}\left(\widehat{\alpha}_{2}-\frac{n-1}{n} s^{2}, \widehat{\alpha}_{1}\right) \\
& =\widehat{\operatorname{Cov}}\left(\widehat{\alpha}_{2}, \widehat{\alpha}_{1}\right)-\frac{n-1}{n} \widehat{\operatorname{Cov}}\left(s^{2}, \widehat{\alpha}_{1}\right) \\
& =\frac{\widehat{\alpha}_{3}-\widehat{\alpha}_{2} \widehat{\alpha}_{1}}{n-1}-\frac{(n-1) \widehat{\mu}_{3}}{n^{2}},
\end{aligned}
$$

and

$$
\widehat{\operatorname{Cov}}\left\{\widehat{\alpha}_{1}, \widehat{V}\left(\widehat{\alpha}_{1}\right)\right\}=\widehat{\operatorname{Cov}}\left(\widehat{\alpha}_{1}, \frac{s^{2}}{n}\right)=\frac{\widehat{\mu}_{3}}{n^{2}} .
$$

From Lemma 3.1:

$$
\begin{aligned}
\widehat{\mu}_{3}= & \widehat{\alpha}_{3}-3 \widehat{\alpha}_{2} \widehat{\alpha}_{1}+3 \frac{\widehat{\alpha}_{3}-\widehat{\alpha}_{2} \widehat{\alpha}_{1}}{n-1}+2 \widehat{\alpha}_{1}^{3} \\
& -2 \widehat{\alpha}_{1} \frac{\widehat{\alpha}_{2}-\widehat{\alpha}_{1}^{2}}{n-1}-2\left\{\frac{\widehat{\alpha}_{3}-\widehat{\alpha}_{2} \widehat{\alpha}_{1}}{n-1}-\frac{(n-1) \widehat{\mu}_{3}}{n^{2}}\right\}+2 \frac{\widehat{\mu}_{3}}{n^{2}},
\end{aligned}
$$

which implies

$$
\widehat{\mu}_{3}=\frac{n^{2}}{n^{2}-3 n+2}\left(\widehat{\alpha}_{3}-3 \widehat{\alpha}_{2} \widehat{\alpha}_{1}+2 \widehat{\alpha}_{1}^{3}\right) .
$$

Moreover, this unbiased estimator, $\widehat{\mu}_{3}$, is invariant under permutations of the sample values $\left(x_{1}, x_{2}, \ldots, x_{n}\right)$. So, $\widehat{\mu}_{3}$ is optimal (UMVU estimator) for the parameter $\mu_{3}$ under distribution-free setting (see [65, page 150]). Hence, the proof. 
Remark 3.1 The estimator, $\widehat{\mu}_{3}$, is unbiased (as it has been stated in Theorem 3.1) and consistent for $\mu_{3}$ as $\widehat{\alpha}_{k}$ converges in probability to $\alpha_{k}(k=1,2,3)$. As $n \rightarrow \infty$,

$$
\widehat{\mu}_{3} \rightarrow \alpha_{3}-3 \alpha_{2} \alpha_{1}+2 \alpha_{1}^{3}=\mu_{3},
$$

i.e., $\widehat{\mu}_{3}$ converges in probability to $\mu_{3}$. In Theorem 3.1 , the estimator, $\widehat{\mu}_{3}$, has a variance which converges to 'zero' as $n \rightarrow \infty$. Following Olkin [38], another consistent estimator of $\mu_{3}$ is:

$$
m_{3}=\widehat{\alpha}_{3}-3 \widehat{\alpha}_{2} \widehat{\alpha}_{1}+2 \widehat{\alpha}_{1}^{3} .
$$

Taking expectations, we obtain

$$
E\left(m_{3} ; \mu_{3}\right)=\mu_{3}+\frac{2-3 n}{n^{2}} \mu_{3}
$$

which shows that $m_{3}$ is biased. For estimating $\mu_{3}$, the bias of $m_{3}$ is given by

$$
B\left(m_{3} ; \mu_{3}\right)=E\left(m_{3} ; \mu_{3}\right)-\mu_{3}=\frac{2-3 n}{n^{2}} \mu_{3},
$$

which converges to 'zero' as $n \rightarrow \infty$. So, $m_{3}$ is asymptotically unbiased for $\mu_{3}$.

Remark 3.2 According to Dodge and Rousson [9], another expression of an optimal unbiased estimator (under distribution-free setting and for estimating $\mu_{3}$ ) is given by

$$
M_{3}=\frac{n \sum_{i=1}^{n}\left(x_{i}-\widehat{\alpha}_{1}\right)^{3}}{(n-1)(n-2)}=\frac{n^{2} m_{3}}{n^{2}-3 n+2},
$$

which coincides with our estimator $\widehat{\mu}_{3}$ deduced in Theorem 3.1 .

Theorem 3.2 The unbiased estimator $\widehat{\mu}_{3}$ (in Lemma 3.1) is also valid for stratified sampling with independent srsr designs in each stratum, where:

$$
\begin{aligned}
& \widehat{\alpha}_{k}=\sum_{h=1}^{L} W_{h} \widehat{\alpha}_{k h}(k=1,2,3) \text { and } \widehat{\alpha}_{k h}=\frac{1}{n_{h}} \sum_{i=1}^{n_{h}} x_{h i}^{k}(h=1,2, \ldots, L), \\
& \widehat{V}\left(\widehat{\alpha}_{1}\right)=\sum_{h=1}^{L} W_{h}^{2} \frac{1}{n_{h}-1}\left(\widehat{\alpha}_{2 h}-\widehat{\alpha}_{1 h}^{2}\right), \\
& \widehat{\operatorname{Cov}}\left(\widehat{\alpha}_{2}, \widehat{\alpha}_{1}\right)=\sum_{h=1}^{L} W_{h}^{2} \frac{1}{n_{h}-1}\left(\widehat{\alpha}_{3 h}-\widehat{\alpha}_{2 h} \widehat{\alpha}_{1 h}\right), \\
& \widehat{\operatorname{Cov}}\left(\widehat{\alpha}_{1}^{2}, \widehat{\alpha}_{1}\right)=\sum_{h=1}^{L} W_{h}^{3}\left\{\frac{\widehat{\alpha}_{3 h}-\widehat{\alpha}_{2 h} \widehat{\alpha}_{1 h}}{n_{h}-1}-\frac{\left(n_{h}-1\right) \widehat{\mu}_{3 h}}{n_{h}^{2}}\right\} \\
& +2 \sum_{h=1}^{L-1} \sum_{g>h}^{L} W_{h} W_{g}\left\{W_{h} \frac{1}{n_{h}-1} \widehat{\alpha}_{1 g}\left(\widehat{\alpha}_{2 h}-\widehat{\alpha}_{1 h}^{2}\right)\right. \\
& \left.+W_{g} \frac{1}{n_{g}-1} \widehat{\alpha}_{1 h}\left(\widehat{\alpha}_{2 g}-\widehat{\alpha}_{1 g}^{2}\right)\right\}
\end{aligned}
$$


and

$$
\widehat{\operatorname{Cov}}\left\{\widehat{\alpha}_{1}, \widehat{V}\left(\widehat{\alpha}_{1}\right)\right\}=\sum_{h=1}^{L} W_{h}^{3} \frac{1}{n_{h}-1} \frac{\widehat{\mu}_{3 h}}{n_{h}},
$$

where

$$
\begin{aligned}
\widehat{\mu}_{3 h} & =\frac{n_{h}^{2}}{n_{h}^{2}-3 n_{h}+2}\left(\widehat{\alpha}_{3 h}-3 \widehat{\alpha}_{2 h} \widehat{\alpha}_{1 h}+2 \widehat{\alpha}_{1 h}^{3}\right)=\frac{n_{h}^{2} m_{3 h}}{n_{h}^{2}-3 n_{h}+2} \\
& =\frac{n_{h} \sum_{i=1}^{n_{h}}\left(x_{h i}-\widehat{\alpha}_{1 h}\right)^{3}}{\left(n_{h}-1\right)\left(n_{h}-2\right)}=M_{3 h} .
\end{aligned}
$$

Proof The stochastic independence (of the realized sampling in two different strata) provides

$$
\begin{gathered}
\widehat{V}\left(\widehat{\alpha}_{1}\right)=\sum_{h=1}^{L} W_{h}^{2} \frac{s_{h}^{2}}{n_{h}}=\sum_{h=1}^{L} W_{h}^{2} \frac{1}{n_{h}-1}\left(\widehat{\alpha}_{2 h}-\widehat{\alpha}_{1 h}^{2}\right), \\
\widehat{\operatorname{Cov}}\left(\widehat{\alpha}_{2}, \widehat{\alpha}_{1}\right)=\sum_{h=1}^{L} W_{h}^{2} \widehat{\operatorname{Cov}}\left(\widehat{\alpha}_{2 h}, \widehat{\alpha}_{1 h}\right)=\sum_{h=1}^{L} W_{h}^{2} \frac{1}{n_{h}-1}\left(\widehat{\alpha}_{3 h}-\widehat{\alpha}_{2 h} \widehat{\alpha}_{1 h}\right),
\end{gathered}
$$

and

$$
\begin{aligned}
\widehat{\operatorname{Cov}}\left(\widehat{\alpha}_{1}^{2}, \widehat{\alpha}_{1}\right)= & \widehat{\operatorname{Cov}}\left\{\left(\sum_{h=1}^{L} W_{h} \widehat{\alpha}_{1 h}\right)^{2}, \sum_{g=1}^{L} W_{g} \widehat{\alpha}_{1 g}\right\} \\
= & \widehat{\operatorname{Cov}}\left(\sum_{h=1}^{L} W_{h}^{2} \widehat{\alpha}_{1 h}^{2}+\sum_{h=1}^{L} \sum_{g \neq h}^{L} W_{h} W_{g} \widehat{\alpha}_{1 h} \widehat{\alpha}_{1 g}, \sum_{k=1}^{L} W_{k} \widehat{\alpha}_{1 k}\right) \\
= & \widehat{\operatorname{Cov}}\left(\sum_{h=1}^{L} W_{h}^{2} \widehat{\alpha}_{1 h}^{2}, \sum_{k=1}^{L} W_{k} \widehat{\alpha}_{1 k}\right) \\
& +\widehat{\operatorname{Cov}}\left(\sum_{h=1}^{L} \sum_{g \neq h}^{L} W_{h} W_{g} \widehat{\alpha}_{1 h} \widehat{\alpha}_{1 g}, \sum_{k=1}^{L} W_{k} \widehat{\alpha}_{1 k}\right),
\end{aligned}
$$

where

$$
\begin{aligned}
\widehat{\operatorname{Cov}} & \left(\sum_{h=1}^{L} W_{h}^{2} \widehat{\alpha}_{1 h}^{2}, \sum_{k=1}^{L} W_{k} \widehat{\alpha}_{1 k}\right) \\
= & \sum_{h=1}^{L} W_{h}^{3} \widehat{\operatorname{Cov}}\left(\widehat{\alpha}_{1 h}^{2}, \widehat{\alpha}_{1 h}\right) \\
= & \sum_{h=1}^{L} W_{h}^{3}\left\{\widehat{\operatorname{Cov}}\left(\widehat{\alpha}_{2 h}, \widehat{\alpha}_{1 h}\right)-\widehat{\operatorname{Cov}}\left(\frac{n_{h}-1}{n_{h}} s_{h}^{2}, \widehat{\alpha}_{1 h}\right)\right\} \\
= & \sum_{h=1}^{L} W_{h}^{3}\left\{\frac{\widehat{\alpha}_{3 h}-\widehat{\alpha}_{2 h} \widehat{\alpha}_{1 h}}{n_{h}-1}-\frac{\left(n_{h}-1\right) \widehat{\mu}_{3 h}}{n_{h}^{2}}\right\}
\end{aligned}
$$


and

$$
\begin{aligned}
\widehat{\operatorname{Cov}} & \left(\sum_{h=1}^{L} \sum_{g \neq h}^{L} W_{h} W_{g} \widehat{\alpha}_{1 h} \widehat{\alpha}_{1 g}, \sum_{k=1}^{L} W_{k} \widehat{\alpha}_{1 k}\right) \\
= & 2 \widehat{\operatorname{Cov}}\left(\sum_{h=1}^{L-1} \sum_{g>h}^{L} W_{h} W_{g} \widehat{\alpha}_{1 h} \widehat{\alpha}_{1 g}, \sum_{k=1}^{L} W_{k} \widehat{\alpha}_{1 k}\right) \\
= & 2 \sum_{h=1}^{L-1} \sum_{g>h}^{L} W_{h} W_{g}\left\{W_{h} \widehat{\operatorname{Cov}}\left(\widehat{\alpha}_{1 h} \widehat{\alpha}_{1 g}, \widehat{\alpha}_{1 h}\right)+W_{g} \widehat{\operatorname{Cov}}\left(\widehat{\alpha}_{1 h} \widehat{\alpha}_{1 g}, \widehat{\alpha}_{1 g}\right)\right\} .
\end{aligned}
$$

We note from Raj [41, Theorem I.8] or alternatively Tucker [58] that

$$
\begin{aligned}
\widehat{\operatorname{Cov}}\left(\widehat{\alpha}_{1 h} \widehat{\alpha}_{1 g}, \widehat{\alpha}_{1 h}\right)= & \widehat{E}\left\{\operatorname{Cov}\left(\widehat{\alpha}_{1 h} \widehat{\alpha}_{1 g}, \widehat{\alpha}_{1 h} \mid \widehat{\alpha}_{1 g}\right)\right\} \\
& +\widehat{\operatorname{Cov}}\left\{E\left(\widehat{\alpha}_{1 h} \widehat{\alpha}_{1 g} \mid \widehat{\alpha}_{1 g}\right), E\left(\widehat{\alpha}_{1 h} \mid \widehat{\alpha}_{1 g}\right)\right\} \\
= & \widehat{E}\left\{\widehat{\alpha}_{1 g} V\left(\widehat{\alpha}_{1 h}\right)\right\} \\
= & \left\{\alpha_{1 g} V\left(\widehat{\alpha}_{1 h}\right)\right\}=\widehat{\alpha}_{1 g} \widehat{V}\left(\widehat{\alpha}_{1 h}\right) \\
= & \widehat{\alpha}_{1 g} \frac{1}{n_{h}-1}\left(\widehat{\alpha}_{2 h}-\widehat{\alpha}_{1 h}^{2}\right)
\end{aligned}
$$

and

$$
\widehat{\operatorname{Cov}}\left(\widehat{\alpha}_{1 h} \widehat{\alpha}_{1 g}, \widehat{\alpha}_{1 g}\right)=\widehat{\alpha}_{1 h} \frac{1}{n_{g}-1}\left(\widehat{\alpha}_{2 g}-\widehat{\alpha}_{1 g}^{2}\right) .
$$

Then,

$$
\begin{aligned}
\widehat{\operatorname{Cov}}\left\{\widehat{\alpha}_{1}, \widehat{V}\left(\widehat{\alpha}_{1}\right)\right\} & =\widehat{\operatorname{Cov}}\left(\sum_{h=1}^{L} W_{h} \widehat{\alpha}_{1 h}, \sum_{g=1}^{L} W_{g}^{2} \frac{s_{g}^{2}}{n_{g}}\right) \\
& =\widehat{\operatorname{Cov}}\left\{\sum_{h=1}^{L} W_{h} \widehat{\alpha}_{1 h}, \sum_{g=1}^{L} W_{g}^{2} \frac{1}{n_{g}-1}\left(\widehat{\alpha}_{2 g}-\widehat{\alpha}_{1 g}^{2}\right)\right\} \\
& =\sum_{h=1}^{L} W_{h}^{3} \frac{1}{n_{h}-1}\left\{\widehat{\operatorname{Cov}}\left(\widehat{\alpha}_{1 h}, \widehat{\alpha}_{2 h}\right)-\widehat{\operatorname{Cov}}\left(\widehat{\alpha}_{1 h}, \widehat{\alpha}_{1 h}^{2}\right)\right\} \\
& =\sum_{h=1}^{L} W_{h}^{3} \frac{\widehat{\mu}_{3 h}}{n_{h}^{2}},
\end{aligned}
$$

where

$$
\widehat{\operatorname{Cov}}\left(\widehat{\alpha}_{1 h}, \widehat{\alpha}_{2 h}\right)=\frac{\widehat{\alpha}_{3 h}-\widehat{\alpha}_{2 h} \widehat{\alpha}_{1 h}}{n_{h}-1}
$$

and

$$
\widehat{\operatorname{Cov}}\left(\widehat{\alpha}_{1 h}, \widehat{\alpha}_{1 h}^{2}\right)=\frac{\widehat{\alpha}_{3 h}-\widehat{\alpha}_{2 h} \widehat{\alpha}_{1 h}}{n_{h}-1}-\frac{\left(n_{h}-1\right) \widehat{\mu}_{3 h}}{n_{h}^{2}} .
$$


So, from Theorem 3.1, we have

$$
\widehat{\mu}_{3 h}=\frac{n_{h}^{2}}{n_{h}^{2}-3 n_{h}+2}\left(\widehat{\alpha}_{3 h}-3 \widehat{\alpha}_{2 h} \widehat{\alpha}_{1 h}+2 \widehat{\alpha}_{1 h}^{3}\right) .
$$

Hence, the proof.

\section{Population central moment of order four}

Our general method (for obtaining optimal unbiased estimators for the population variance, $\sigma^{2}$, or for the population central moment of order three) is also useful in investigating UMVU estimators for the fourth population central moment. Some relevant references in this context are Polo [39], Ruiz Espejo and Ruiz Espejo [46] and Maritz [33].

The fourth population central moment is defined by

$$
\mu_{4}=\alpha_{4}-4 \alpha_{3} \alpha_{1}+6 \alpha_{2} \alpha_{1}^{2}-3 \alpha_{1}^{4}=\int_{-\infty}^{\infty}(x-\mu)^{4} d F(x)(<\infty),
$$

where $\mu=\alpha_{1}$ is the population mean, and $F(x)$ is the distribution function of the random variable $X$.

The key result of this section is the UMVU estimator of the parameter $\mu_{4}$ in (1) under a distribution-free setting and a concrete sampling design. An application is provided for variance estimation of the 'sample quasivariance' $s^{2}$ defined by

$$
s^{2}=\frac{n}{n-1}\left(\widehat{\alpha}_{2}-\widehat{\alpha}_{1}^{2}\right) \text {. }
$$

Lemma 4.1 If $\widehat{\omega}$ denotes an unbiased estimator of $\omega$ then an unbiased estimator of $\mu_{4}$ (for any sampling design) is

$$
\begin{aligned}
\widehat{\mu}_{4}= & \widehat{\alpha}_{4}-4 \widehat{\alpha}_{3} \widehat{\alpha}_{1}+4 \widehat{\operatorname{Cov}}\left(\widehat{\alpha}_{3}, \widehat{\alpha}_{1}\right)+6 \widehat{\alpha}_{2} \widehat{\alpha_{1}^{2}}-6 \widehat{V}\left(\widehat{\alpha}_{2}\right) \\
& +6 \widehat{\operatorname{Cov}}\left(\widehat{\alpha}_{2}, s^{2}\right)-3 \widehat{\alpha}_{1}^{4}+3 \widehat{V}\left(\widehat{\alpha}_{1}^{2}\right)+3\left[\left\{\widehat{V\left(\widehat{\alpha}_{1}\right)}\right\}^{2}\right]+6\left\{\alpha_{1}^{2} V\left(\widehat{\alpha}_{1}\right)\right\} .
\end{aligned}
$$

Proof We have

$$
E\left(\widehat{\alpha}_{4}\right)=\alpha_{4}
$$

and

$$
E\left(-4 \widehat{\alpha}_{3} \widehat{\alpha}_{1}\right)=-4 E\left(\widehat{\alpha}_{3} \widehat{\alpha}_{1}\right)=-4\left\{\operatorname{Cov}\left(\widehat{\alpha}_{3}, \widehat{\alpha}_{1}\right)+\alpha_{3} \alpha_{1}\right\}
$$

which implies

$$
\left(\widehat{-4 \alpha_{3} \alpha_{1}}\right)=-4 \widehat{\left(\alpha_{3} \alpha_{1}\right)}=-4\left\{\widehat{\alpha}_{3} \widehat{\alpha}_{1}-\widehat{\operatorname{Cov}}\left(\widehat{\alpha}_{3}, \widehat{\alpha}_{1}\right)\right\}
$$

Also,

$$
\begin{aligned}
E\left(\widehat{\alpha}_{2} \widehat{\alpha}_{1}^{2}\right) & =E\left\{\widehat{\alpha}_{2}\left(\widehat{\alpha}_{2}-s^{2}\right)\right\} \\
& =E\left(\widehat{\alpha}_{2}^{2}-\widehat{\alpha}_{2} s^{2}\right)=E\left(\widehat{\alpha}_{2}^{2}\right)-E\left(\widehat{\alpha}_{2} s^{2}\right) \\
& =\left\{V\left(\widehat{\alpha}_{2}\right)+\alpha_{2}^{2}\right\}-\left\{\operatorname{Cov}\left(\widehat{\alpha}_{2}, s^{2}\right)+\alpha_{2} \sigma^{2}\right\} \\
& =\alpha_{2}\left(\alpha_{2}-\sigma^{2}\right)+V\left(\widehat{\alpha}_{2}\right)-\operatorname{Cov}\left(\widehat{\alpha}_{2}, s^{2}\right) \\
& =\alpha_{2} \alpha_{1}^{2}+V\left(\widehat{\alpha}_{2}\right)-\operatorname{Cov}\left(\widehat{\alpha}_{2}, s^{2}\right),
\end{aligned}
$$


or

$$
\alpha_{2} \alpha_{1}^{2}=E\left(\widehat{\alpha_{2}} \widehat{\alpha_{1}^{2}}\right)-V\left(\widehat{\alpha}_{2}\right)+\operatorname{Cov}\left(\widehat{\alpha}_{2}, s^{2}\right)
$$

gives

$$
\left(\widehat{6 \alpha_{2} \alpha_{1}^{2}}\right)=6 \widehat{\alpha}_{2} \widehat{\alpha_{1}^{2}}-6 \widehat{V}\left(\widehat{\alpha}_{2}\right)+6 \widehat{\operatorname{Cov}}\left(\widehat{\alpha}_{2}, s^{2}\right) .
$$

We have

$$
\begin{aligned}
E\left(\widehat{\alpha}_{1}^{4}\right) & =E\left(\widehat{\alpha}_{1}^{2} \widehat{\alpha}_{1}^{2}\right) \\
& =V\left(\widehat{\alpha}_{1}^{2}\right)+\left\{E\left(\widehat{\alpha}_{1}^{2}\right)\right\}^{2} \\
& =V\left(\widehat{\alpha}_{1}^{2}\right)+\left\{V\left(\widehat{\alpha}_{1}\right)+\alpha_{1}^{2}\right\}^{2} \\
& =V\left(\widehat{\alpha}_{1}^{2}\right)+\left\{V\left(\widehat{\alpha}_{1}\right)\right\}^{2}+\alpha_{1}^{4}+2 \alpha_{1}^{2} V\left(\widehat{\alpha}_{1}\right),
\end{aligned}
$$

or

$$
\alpha_{1}^{4}=E\left(\widehat{\alpha}_{1}^{4}\right)-V\left(\widehat{\alpha}_{1}^{2}\right)-\left\{V\left(\widehat{\alpha}_{1}\right)\right\}^{2}-2 \alpha_{1}^{2} V\left(\widehat{\alpha}_{1}\right)
$$

So,

$$
\widehat{\left(-3 \alpha_{1}^{4}\right)}=-3 \widehat{\alpha}_{1}^{4}+3 \widehat{V}\left(\widehat{\alpha}_{1}^{2}\right)+3\left[\left\{\widehat{V\left(\widehat{\alpha}_{1}\right)}\right\}^{2}\right]+6\left\{\alpha_{1}^{2} V\left(\widehat{\alpha}_{1}\right)\right\} .
$$

Hence, the unbiased estimator of $\mu_{4}$ is

$$
\widehat{\mu}_{4}=\widehat{\alpha}_{4}+\left(\widehat{-4 \alpha_{3} \alpha_{1}}\right)+\left(\widehat{6 \alpha_{2} \alpha_{1}^{2}}\right)+\left(\widehat{-3 \alpha_{1}^{4}}\right),
$$

where $\widehat{\alpha}_{4},\left(\widehat{-4 \alpha_{3} \alpha_{1}}\right),\left(\widehat{6 \alpha_{2} \alpha_{1}^{2}}\right)$ and $\left(\widehat{-3 \alpha_{1}^{4}}\right)$ are given by (2), (3), (4) and (5), respectively. This proves Lemma 4.1 .

Lemma 4.2 For simple random sampling designs with replacement of size $n$ (srsr-n),

$$
\widehat{\operatorname{Cov}}\left(\widehat{\alpha}_{3}, \widehat{\alpha}_{1}\right)=\frac{\widehat{\alpha}_{4}-\widehat{\alpha}_{3} \widehat{\alpha}_{1}}{n-1} \text {. }
$$

Proof We have

$$
\begin{aligned}
\operatorname{Cov}\left(\widehat{\alpha}_{3}, \widehat{\alpha}_{1}\right) & =E\left(\widehat{\alpha}_{3} \widehat{\alpha}_{1}\right)-\alpha_{3} \alpha_{1} \\
& =\frac{1}{n}\left\{\alpha_{4}+(n-1) \alpha_{3} \alpha_{1}\right\}-\alpha_{3} \alpha_{1} \\
& =\frac{\alpha_{4}-\alpha_{3} \alpha_{1}}{n} .
\end{aligned}
$$

So, an unbiased estimator of $\operatorname{Cov}\left(\widehat{\alpha}_{3}, \widehat{\alpha}_{1}\right)$ is

$$
\begin{aligned}
\widehat{\operatorname{Cov}}\left(\widehat{\alpha}_{3}, \widehat{\alpha}_{1}\right) & =\frac{\left(\alpha_{4}-\widehat{\alpha_{3}} \alpha_{1}\right)}{n}=\frac{\left.\widehat{\alpha}_{4}-\widehat{\left(\alpha_{3} \alpha_{1}\right.}\right)}{n} \\
& =\frac{\widehat{\alpha}_{4}}{n}-\frac{\widehat{\alpha_{3}} \widehat{\alpha}_{1}-\widehat{\operatorname{Cov}}\left(\widehat{\alpha}_{3}, \widehat{\alpha}_{1}\right)}{n} \\
& =\frac{\widehat{\alpha}_{4}-\widehat{\alpha}_{3} \widehat{\alpha}_{1}}{n}+\frac{\widehat{\operatorname{Cov}}\left(\widehat{\alpha}_{3}, \widehat{\alpha}_{1}\right)}{n}
\end{aligned}
$$


or

$$
\widehat{\operatorname{Cov}}\left(\widehat{\alpha}_{3}, \widehat{\alpha}_{1}\right)=\frac{\widehat{\alpha}_{4}-\widehat{\alpha}_{3} \widehat{\alpha}_{1}}{n-1},
$$

which proves Lemma 4.2.

Lemma 4.3 For srsr-n designs,

$$
\widehat{V}\left(\widehat{\alpha}_{2}\right)=\frac{\widehat{\alpha}_{4}-\widehat{\alpha}_{2}^{2}}{n-1} .
$$

Proof We note that

$$
V\left(\widehat{\alpha}_{2}\right)=E\left(\widehat{\alpha}_{2}^{2}\right)-\left\{E\left(\widehat{\alpha}_{2}\right)\right\}^{2}=\frac{\alpha_{4}-\alpha_{2}^{2}}{n} .
$$

So, an unbiased estimator of $V\left(\widehat{\alpha}_{2}\right)$ is

$$
\widehat{V}\left(\widehat{\alpha}_{2}\right)=\frac{\widehat{\alpha}_{4}-\widehat{\alpha_{2}^{2}}}{n}=\frac{\widehat{\alpha}_{4}-\left\{\widehat{\alpha}_{2}^{2}-\widehat{V}\left(\widehat{\alpha}_{2}\right)\right\}}{n}=\frac{\widehat{\alpha}_{4}-\widehat{\alpha}_{2}^{2}}{n}+\frac{\widehat{V}\left(\widehat{\alpha}_{2}\right)}{n}
$$

or

$$
\widehat{V}\left(\widehat{\alpha}_{2}\right)=\frac{\widehat{\alpha}_{4}-\widehat{\alpha}_{2}^{2}}{n-1} .
$$

Hence, Lemma 4.3 is proved.

Lemma 4.4 For srsr-n designs,

$$
\widehat{\operatorname{Cov}}\left(\widehat{\alpha}_{2}, s^{2}\right)=\frac{\widehat{\alpha}_{4}-\widehat{\alpha}_{2}^{2}}{n-1}+\frac{2}{n-2} \widehat{\alpha}_{2}\left(\widehat{\alpha}_{2}-s^{2}\right)-\frac{2\left(n \widehat{\alpha}_{3} \widehat{\alpha}_{1}-\widehat{\alpha}_{4}\right)}{(n-1)(n-2)} .
$$

Proof We have

$$
\operatorname{Cov}\left(\widehat{\alpha}_{2}, s^{2}\right)=E\left(\widehat{\alpha}_{2} s^{2}\right)-E\left(\widehat{\alpha}_{2}\right) E\left(s^{2}\right)=\frac{1}{n}\left(\alpha_{4}-\alpha_{2}^{2}-2 \alpha_{1} \alpha_{3}+2 \alpha_{2} \alpha_{1}^{2}\right) .
$$

So, the unbiased estimator of $\operatorname{Cov}\left(\widehat{\alpha}_{2}, s^{2}\right)$ is

$$
\begin{aligned}
\widehat{\operatorname{Cov}}\left(\widehat{\alpha}_{2}, s^{2}\right)= & \frac{1}{n}\left(\alpha_{4}-\alpha_{2}^{2}-\widehat{2 \alpha_{1} \alpha_{3}}+2 \alpha_{2} \alpha_{1}^{2}\right) \\
= & \frac{1}{n}\left(\widehat{\alpha_{4}-\alpha_{2}^{2}}\right)+\frac{2}{n}\left(\alpha_{2} \alpha_{1}^{2}-\alpha_{1} \alpha_{3}\right) \\
= & \left.\frac{\widehat{\alpha}_{4}-\widehat{\alpha}_{2}^{2}}{n-1}+\frac{2}{n}\left\{\widehat{\left(\alpha_{2} \alpha_{1}^{2}\right.}\right)-\widehat{\left(\alpha_{1} \alpha_{3}\right)}\right\} \\
= & \frac{\widehat{\alpha}_{4}-\widehat{\alpha}_{2}^{2}}{n-1}+\frac{2}{n}\left[\left\{\left(\widehat{\alpha}_{2}-s^{2}\right) \widehat{\alpha}_{2}-\frac{\widehat{\alpha}_{4}-\widehat{\alpha}_{2}^{2}}{n-1}+\widehat{\operatorname{Cov}}\left(\widehat{\alpha}_{2}, s^{2}\right)\right\}\right. \\
& \left.-\frac{n \widehat{\alpha}_{3} \widehat{\alpha}_{1}-\widehat{\alpha}_{4}}{n-1}\right]
\end{aligned}
$$


since

$$
\begin{aligned}
\widehat{\left(\alpha_{2} \alpha_{1}^{2}\right)} & =\widehat{\alpha}_{2} \widehat{\alpha_{1}^{2}}-\widehat{\operatorname{Cov}}\left(\widehat{\alpha}_{2}, \widehat{\alpha_{1}^{2}}\right) \\
& =\widehat{\alpha}_{2}\left(\widehat{\alpha}_{2}-s^{2}\right)-\left\{\widehat{V}\left(\widehat{\alpha}_{2}\right)-\widehat{\operatorname{Cov}}\left(\widehat{\alpha}_{2}, s^{2}\right)\right\} \\
& =\widehat{\alpha}_{2}\left(\widehat{\alpha}_{2}-s^{2}\right)-\frac{\widehat{\alpha}_{4}-\widehat{\alpha}_{2}^{2}}{n-1}+\widehat{\operatorname{Cov}}\left(\widehat{\alpha}_{2}, s^{2}\right),
\end{aligned}
$$

and

$$
\widehat{\left(\alpha_{1} \alpha_{3}\right)}=\widehat{\alpha}_{1} \widehat{\alpha}_{3}-\widehat{\operatorname{Cov}}\left(\widehat{\alpha}_{3}, \widehat{\alpha}_{1}\right)=\widehat{\alpha}_{3} \widehat{\alpha}_{1}-\frac{\widehat{\alpha}_{4}-\widehat{\alpha}_{3} \widehat{\alpha}_{1}}{n-1}=\frac{n \widehat{\alpha}_{3} \widehat{\alpha}_{1}-\widehat{\alpha}_{4}}{n-1} .
$$

It follows from (6) that

$$
\widehat{\operatorname{Cov}}\left(\widehat{\alpha}_{2}, s^{2}\right)\left(1-\frac{2}{n}\right)=\frac{\widehat{\alpha}_{4}-\widehat{\alpha}_{2}^{2}}{n-1}\left(1-\frac{2}{n}\right)+\frac{2}{n} \widehat{\alpha}_{2}\left(\widehat{\alpha}_{2}-s^{2}\right)-\frac{2\left(n \widehat{\alpha}_{3} \widehat{\alpha}_{1}-\widehat{\alpha}_{4}\right)}{n(n-1)},
$$

or

$$
\widehat{\operatorname{Cov}}\left(\widehat{\alpha}_{2}, s^{2}\right)=\frac{\widehat{\alpha}_{4}-\widehat{\alpha}_{2}^{2}}{n-1}+\frac{2}{n-2} \widehat{\alpha}_{2}\left(\widehat{\alpha}_{2}-s^{2}\right)-\frac{2\left(n \widehat{\alpha}_{3} \widehat{\alpha}_{1}-\widehat{\alpha}_{4}\right)}{(n-1)(n-2)} .
$$

So, Lemma 4.4 is proved.

Lemma 4.5 For srsr-n designs,

$$
\widehat{V}\left(\widehat{\alpha}_{1}^{2}\right)=\frac{\widehat{\alpha}_{4}-\widehat{\alpha}_{2}^{2}}{n-1}+\left(\frac{n-1}{n}\right)^{2} \widehat{V}\left(s^{2}\right)-2 \frac{n-1}{n} \widehat{\operatorname{Cov}}\left(\widehat{\alpha}_{2}, s^{2}\right) .
$$

Proof We have

$$
\begin{aligned}
V\left(\widehat{\alpha}_{1}^{2}\right) & =V\left(\widehat{\alpha}_{2}-\frac{n-1}{n} s^{2}\right) \\
& =V\left(\widehat{\alpha}_{2}\right)+\left(\frac{n-1}{n}\right)^{2} V\left(s^{2}\right)-2 \frac{n-1}{n} \operatorname{Cov}\left(\widehat{\alpha}_{2}, s^{2}\right) .
\end{aligned}
$$

It is well known that

$$
V\left(s^{2}\right)=\frac{\mu_{4}}{n}-\frac{(n-3) \mu_{2}^{2}}{n(n-1)} .
$$

Then an unbiased estimator of $V\left(\widehat{\alpha}_{1}^{2}\right)$ is

$$
\begin{aligned}
\widehat{V}\left(\widehat{\alpha}_{1}^{2}\right) & =\widehat{V}\left(\widehat{\alpha}_{2}-\frac{n-1}{n} s^{2}\right) \\
& =\widehat{V}\left(\widehat{\alpha}_{2}\right)+\left(\frac{n-1}{n}\right)^{2} \widehat{V}\left(s^{2}\right)-2 \frac{n-1}{n} \widehat{\operatorname{Cov}}\left(\widehat{\alpha}_{2}, s^{2}\right),
\end{aligned}
$$

where

$$
\widehat{V}\left(\widehat{\alpha}_{2}\right)=\frac{\widehat{\alpha}_{4}-\widehat{\alpha}_{2}^{2}}{n-1}
$$


An unbiased estimator of $V\left(s^{2}\right)$ is

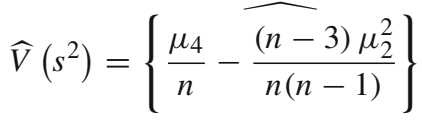

$$
\begin{aligned}
& =\frac{\widehat{\mu}_{4}}{n}-\frac{n-3}{n(n-1)} \widehat{\mu_{2}^{2}} \\
& =\frac{\widehat{\mu}_{4}}{n}-\frac{n-3}{n(n-1)}\left\{s^{4}-\widehat{V}\left(s^{2}\right)\right\},
\end{aligned}
$$

or

$$
\widehat{V}\left(s^{2}\right)=\frac{n-1}{n^{2}-2 n+3} \widehat{\mu}_{4}-\frac{n-3}{n^{2}-2 n+3} s^{4},
$$

where $\widehat{\mu}_{4}$ is the unbiased estimator of $\mu_{4}$ given in Lemma 4.1. By Lemma 4.4, we have $\widehat{\operatorname{Cov}}\left(\widehat{\alpha}_{2}, s^{2}\right)$, and so we obtain the unbiased estimator of the variance of $\widehat{\alpha}_{1}^{2}$. Hence, Lemma 4.5 is proved.

Lemma 4.6 For srsr-n designs,

$$
\left[\left\{\widehat{V\left(\widehat{\alpha}_{1}\right)}\right\}^{2}\right]=\frac{n-1}{n\left(n^{2}-2 n+3\right)} s^{4}-\frac{n-1}{n^{2}\left(n^{2}-2 n+3\right)} \widehat{\mu}_{4} .
$$

Proof It is well known for $s r s r-n$ designs that

$$
V\left(\widehat{\alpha}_{1}\right)=\frac{\mu_{2}}{n} .
$$

Squaring both sides of (8), we obtain

$$
\left\{V\left(\widehat{\alpha}_{1}\right)\right\}^{2}=\frac{\mu_{2}^{2}}{n^{2}} .
$$

Its unbiased estimator is

$$
\left[\left\{\widehat{V\left(\widehat{\alpha}_{1}\right)}\right\}^{2}\right]=\frac{\widehat{\mu_{2}^{2}}}{n^{2}}=\frac{\widehat{\mu}_{2}^{2}-\widehat{V}\left(\widehat{\mu}_{2}\right)}{n^{2}}=\frac{s^{4}-\widehat{V}\left(s^{2}\right)}{n^{2}},
$$

where $\widehat{V}\left(s^{2}\right)$ is given by (7). So, Lemma 4.6 is proved.

Lemma 4.7 For srsr-n designs,

$$
\left\{\widehat{\alpha_{1}^{2} V\left(\widehat{\alpha}_{1}\right)}\right\}=\left(\widehat{\alpha}_{2}-s^{2}\right) \frac{s^{2}}{n}-\frac{1}{n} \widehat{\operatorname{Cov}}\left(\widehat{\alpha}_{2}, s^{2}\right)+\frac{1}{n} \widehat{V}\left(s^{2}\right) .
$$

Proof We have

$$
\alpha_{1}^{2} V\left(\widehat{\alpha}_{1}\right)=\alpha_{1}^{2} \frac{\mu_{2}}{n} .
$$

So, an unbiased estimator of $\alpha_{1}^{2} V\left(\widehat{\alpha}_{1}\right)$ is

$$
\begin{aligned}
& \left.\left\{\widehat{\alpha_{1}^{2} V\left(\widehat{\alpha}_{1}\right.}\right)\right\}=\left(\widehat{\alpha_{1}^{2} \frac{\mu_{2}}{n}}\right)=\widehat{\alpha_{1}^{2}} \frac{\widehat{\mu}_{2}}{n}-\widehat{\operatorname{Cov}}\left(\widehat{\alpha_{1}^{2}}, \frac{\widehat{\mu}_{2}}{n}\right) \\
& =\left(\widehat{\alpha_{2}-\mu_{2}}\right) \frac{\widehat{\mu}_{2}}{n}-\widehat{\operatorname{Cov}}\left\{\left(\widehat{\alpha_{2}-\mu_{2}}\right), \frac{\widehat{\mu}_{2}}{n}\right\} \\
& =\left(\widehat{\alpha}_{2}-s^{2}\right) \frac{s^{2}}{n}-\frac{1}{n} \widehat{\operatorname{Cov}}\left(\widehat{\alpha}_{2}, s^{2}\right)+\frac{1}{n} \widehat{V}\left(s^{2}\right),
\end{aligned}
$$


where $\widehat{\operatorname{Cov}}\left(\widehat{\alpha}_{2}, s^{2}\right)$ is provided in Lemma 4.4, and $\widehat{V}\left(s^{2}\right)$ is provided in (7). Hence, Lemma 4.7 is proved.

Theorem 4.1 For srsr designs of size $n>2$, an optimal unbiased estimator of $\mu_{4}$ (under distribution-free setting) is

$$
\begin{aligned}
\widehat{\mu}_{4}= & \frac{-3 n^{4} \widehat{\alpha}_{1}^{4}+6 n^{4} \widehat{\alpha}_{1}^{2} \widehat{\alpha}_{2}+(9-6 n) n^{2} \widehat{\alpha}_{2}^{2}+\left(-12+8 n-4 n^{2}\right) n^{2} \widehat{\alpha}_{1} \widehat{\alpha}_{3}}{(-3+n)(-2+n)(-1+n) n} \\
& +\frac{\left(3 n-2 n^{2}+n^{3}\right) n \widehat{\alpha}_{4}}{(-3+n)(-2+n)(-1+n) n} .
\end{aligned}
$$

Proof Combining the results of Lemmas 4.1, 4.2, 4.3, 4.4, 4.5 and 4.6, we obtain the result of Theorem 4.1 after simplification. The unbiasedness of $\widehat{\mu}_{4}$ is obvious from Lemma 4.1. The optimality follows from Zacks [65, page 150] because the estimator, $\widehat{\mu}_{4}$, is invariant under permutations of $\left(x_{1}, x_{2}, \ldots, x_{n}\right)$, a simple random sample drawn with replacement.

Remark 4.1 An alternative optimal unbiased estimator of $\mu_{4}$ is given as follows. We have

$$
\left(x_{i}-x_{j}\right)^{4}=x_{i}^{4}-4 x_{i}^{3} x_{j}+6 x_{i}^{2} x_{j}^{2}-4 x_{i} x_{j}^{3}+x_{j}^{4} \quad i \neq j .
$$

Taking expectations of both sides in (9), we obtain

$$
\begin{aligned}
E\left\{\left(x_{i}-x_{j}\right)^{4}\right\} & =\alpha_{4}-4 \alpha_{3} \alpha_{1}+6 \alpha_{2}^{2}-4 \alpha_{1} \alpha_{3}+\alpha_{4} \\
& =2 \alpha_{4}-8 \alpha_{3} \alpha_{1}+6 \alpha_{2}^{2} .
\end{aligned}
$$

It is also known that

$$
\sigma^{4}=\alpha_{2}^{2}-2 \alpha_{2} \alpha_{1}^{2}+\alpha_{1}^{4}
$$

Using (1), (10) and (11), we obtain

$$
\begin{aligned}
E\left\{\frac{1}{2}\left(x_{i}-x_{j}\right)^{4}\right\} & =\alpha_{4}-4 \alpha_{3} \alpha_{1}+3 \alpha_{2}^{2} \\
& =\mu_{4}+3 \alpha_{2}^{2}-6 \alpha_{2} \alpha_{1}^{2}+3 \alpha_{1}^{4}=\mu_{4}+3 \sigma^{4}
\end{aligned}
$$

for $i \neq j$. Now, define

$$
t=\frac{1}{2 n(n-1)} \sum_{i=1}^{n} \sum_{j=1}^{n}\left(x_{i}-x_{j}\right)^{4}=\frac{n}{n-1}\left\{m_{4}+3\left(\frac{n-1}{n} s^{2}\right)^{2}\right\},
$$

where

$$
m_{4}=\frac{1}{n} \sum_{i=1}^{n}\left(x_{i}-\widehat{\alpha}_{1}\right)^{4}
$$

It follows from (12) and (13) that

$$
E(t)=\mu_{4}+3 \sigma^{4} .
$$

Further, from (10) and (13), we have

$$
t=\frac{n}{n-1}\left(\widehat{\alpha}_{4}-4 \widehat{\alpha}_{3} \widehat{\alpha}_{1}+3 \widehat{\alpha}_{2}^{2}\right)=\frac{n}{n-1}\left\{m_{4}+3\left(\frac{n-1}{n} s^{2}\right)^{2}\right\} .
$$


Also,

$$
E\left(s^{4}\right)=V\left(s^{2}\right)+\left\{E\left(s^{2}\right)\right\}^{2}=\frac{\mu_{4}}{n}-\frac{n-3}{n(n-1)} \sigma^{4}+\sigma^{4}=\frac{\mu_{4}}{n}+c \sigma^{4}
$$

implies

$$
\sigma^{4}=\frac{1}{c}\left\{E\left(s^{4}\right)-\frac{\mu_{4}}{n}\right\}
$$

where

$$
c=\frac{n^{2}-2 n+3}{n(n-1)} .
$$

Moreover, we have from (14), (15) and (16) that

$$
E\left(\frac{n}{n-1} m_{4}+3 \frac{n-1}{n} s^{4}\right)=E(t)=\mu_{4}+3 \sigma^{4}=\mu_{4}+\frac{3}{c}\left\{E\left(s^{4}\right)-\frac{\mu_{4}}{n}\right\},
$$

or

$$
E\left(\frac{n}{n-1} m_{4}+3 \frac{n-1}{n} s^{4}\right)=\mu_{4}\left(1-\frac{3}{c n}\right)+\frac{3}{c} E\left(s^{4}\right) .
$$

Hence, from (17), we obtain

$$
\widehat{\mu}_{4}=\left(1-\frac{3}{c n}\right)^{-1}\left\{\frac{n}{n-1} m_{4}+3\left(\frac{n-1}{n}-\frac{1}{c}\right) s^{4}\right\},
$$

the required result.

Remark 4.2 Using Fisher's $k$-statistic, it is possible to obtain another optimal unbiased estimator of $\mu_{4}$. We have that

$$
\mu_{4}=\kappa_{4}+3 \mu_{2}^{2}
$$

where $\kappa_{4}$ is the fourth cumulant. An unbiased estimator of $\kappa_{4}$ is given by $k_{4}$, the corresponding $k$-statistic, and

$$
E\left(k_{2}^{2}\right)=\frac{\mu_{4}}{n}+\frac{n^{2}-2 n+3}{n(n-1)} \mu_{2}^{2} .
$$

So,

$$
\widehat{\mu}_{4}=\frac{n^{2}-2 n+3}{n(n+1)} k_{4}+\frac{3(n-1)}{n+1} k_{2}^{2}
$$

is an unbiased estimator of $\mu_{4}$. As the $k$-statistic can be expressed in terms of power sums $\sum_{i=1}^{n} x_{i}^{r}$ (see [56]), the estimator (18) is invariant under permutations of $\left(x_{1}, x_{2}, \ldots, x_{n}\right)$ and so an UMVU (optimal unbiased estimator) for $\mu_{4}$.

Remark 4.3 Using augmented symmetric functions to evaluate $E\left(\widehat{\alpha}_{4}\right), E\left(\widehat{\alpha}_{3} \widehat{\alpha}_{1}\right)$, etc in

$$
\widehat{\mu}_{4}=c_{1} \widehat{\alpha}_{4}+c_{2} \widehat{\alpha}_{3} \widehat{\alpha}_{1}+c_{3} \widehat{\alpha}_{2}^{2}+c_{4} \widehat{\alpha}_{2} \widehat{\alpha}_{1}^{2}+c_{5} \widehat{\alpha}_{1}^{4},
$$

and collecting terms and using

$$
\mu_{4}=\alpha_{4}-4 \alpha_{3} \alpha_{1}+6 \alpha_{2} \alpha_{1}^{2}-3 \alpha_{1}^{4}
$$


to obtain the coefficients $c_{1}, c_{2}, \ldots, c_{5}$, another optimal unbiased estimator of $\mu_{4}$ can be obtained as

$$
\widehat{\mu}_{4}=\frac{n\left\{\left(n^{2}-2 n+3\right)\left(\widehat{\alpha}_{4}-4 \widehat{\alpha}_{3} \widehat{\alpha}_{1}\right)-3(2 n-3) \widehat{\alpha}_{2}^{2}+3 n^{2}\left(2 \widehat{\alpha}_{2} \widehat{\alpha}_{1}^{2}-\widehat{\alpha}_{1}^{4}\right)\right\}}{(n-1)(n-2)(n-3)} .
$$

Remark 4.4 We have proved that the 'sample quasivariance' $s^{2}$ is an optimal unbiased estimator of the population variance $\sigma^{2}$ under distribution-free setting and $s r s r$ designs. Its variance is

$$
V\left(s^{2}\right)=\frac{\mu_{4}}{n}-\frac{(n-3) \sigma^{4}}{n(n-1)} .
$$

So, an optimal unbiased estimator of $V\left(s^{2}\right)$ is

$$
\widehat{V}\left(s^{2}\right)=\frac{n-1}{n^{2}-2 n+3} \widehat{\mu}_{4}-\frac{n-3}{n^{2}-2 n+3} s^{4},
$$

as in (7), where $\widehat{\mu}_{4}$ is given by Theorem 4.1, or Remarks 4.1, 4.2 and 4.3.

\section{Computer programs}

Here, we give simple functions in $\mathrm{R}$ for computing the unbiased estimators given by Theorems 2.1, 3.1, 3.2 and 4.1. There are five functions in total. The electronic version of these functions can be obtained by contacting the corresponding author.

The following function computes the unbiased estimator of the second central moment given by Theorem 2.1 for pgvps designs. The input parameters needed are $L, N, n_{h}, h=$ $1,2, \ldots, L, W_{h}, h=1,2, \ldots, L, x_{h i}, h=1,2, \ldots, L, i=1,2, \ldots, n_{h}$ and $M_{h i}, h=$ $1,2, \ldots, L, i=1,2, \ldots, n_{h}$.






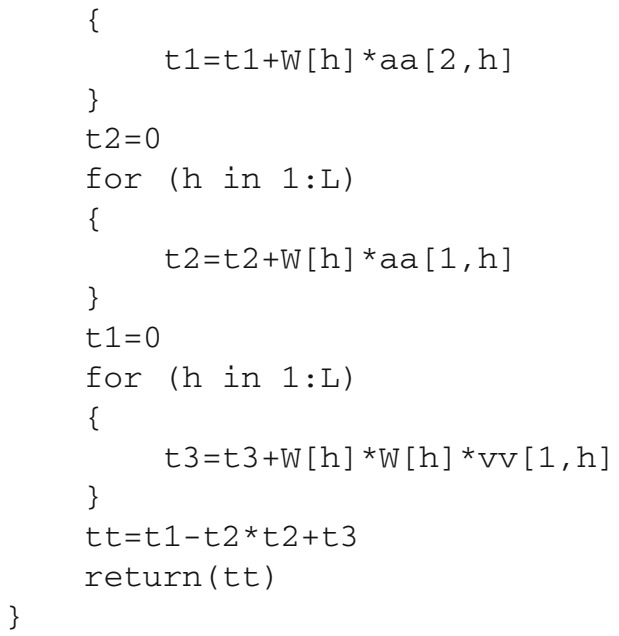

The following function computes the unbiased estimator of the second central moment given by Theorem 2.1 for ppsr designs. The input parameters needed are $L, N, n_{h}, h=$ $1,2, \ldots, L, W_{h}, h=1,2, \ldots, L, x_{h i}, h=1,2, \ldots, L, i=1,2, \ldots, n_{h}$ and $P_{h i}, h=$ $1,2, \ldots, L, i=1,2, \ldots, n_{h}$.

th22=function $(\mathrm{L}, \mathrm{N}, \mathrm{n}, \mathrm{W}, \mathrm{x}, \mathrm{P})$

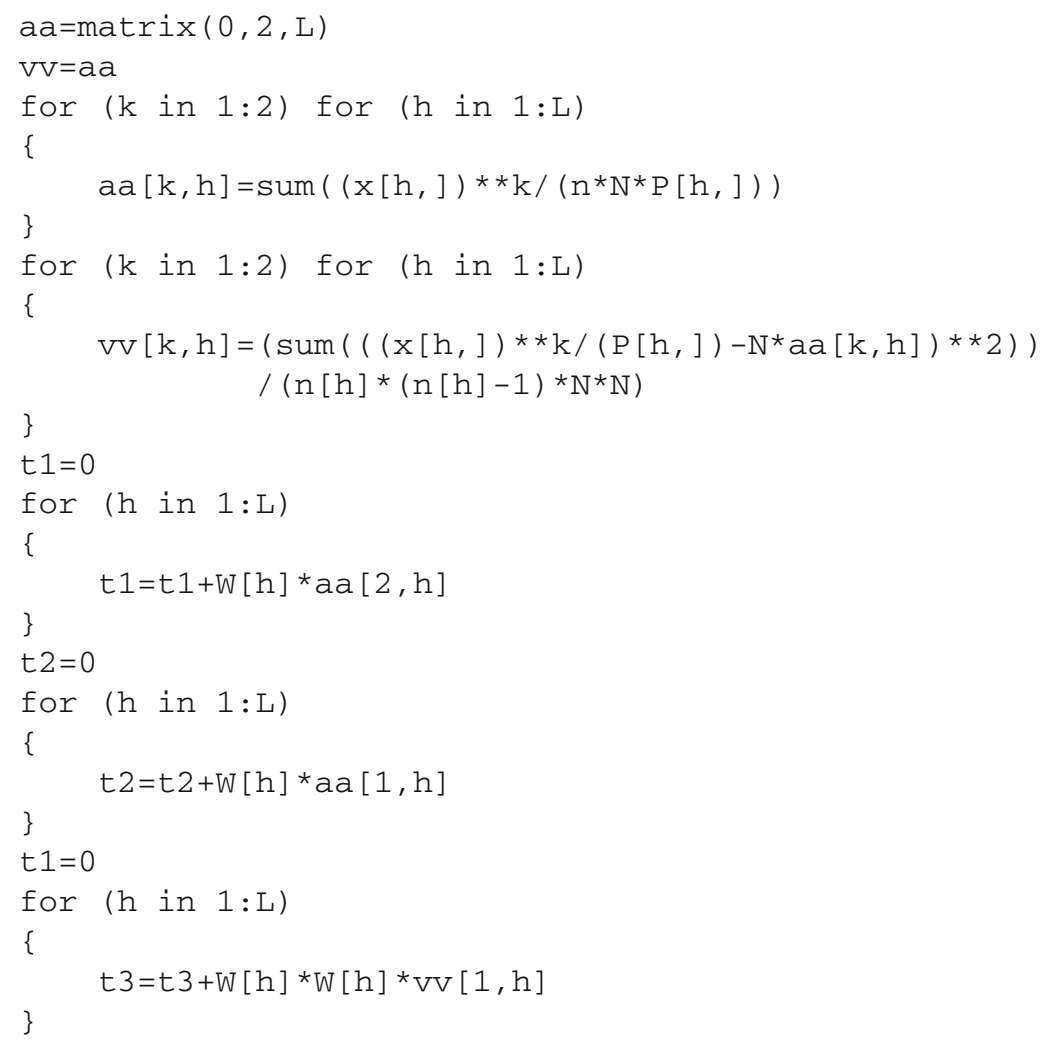




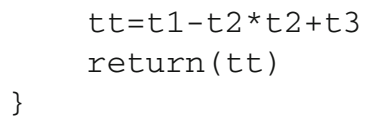

The following function computes the unbiased estimator of the third central moment given by Theorem 3.1. The input parameters needed are $n$ and $x_{i}, i=1,2, \ldots, n$.

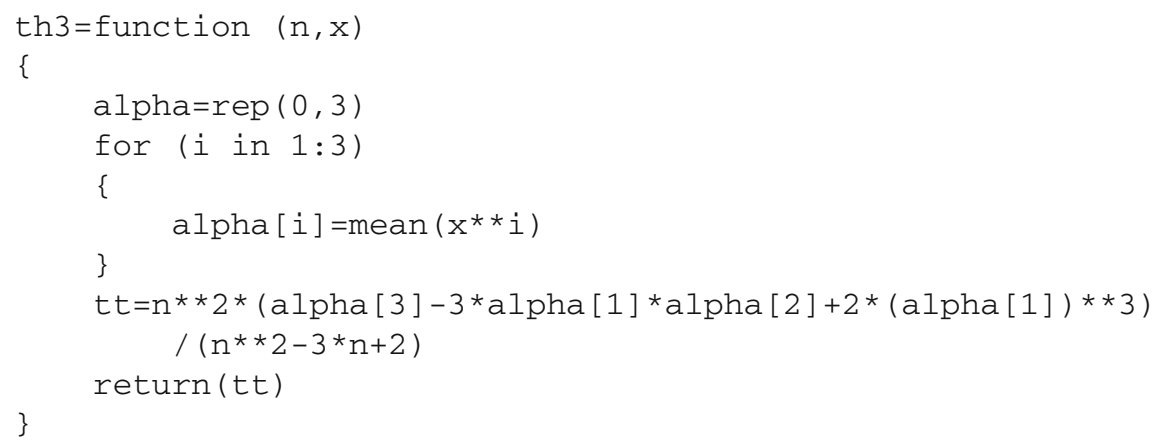

The following function computes the unbiased estimator of the third central moment given by Theorem 3.2. The input parameters needed are $L, n_{h}, h=1,2, \ldots, L, W_{h}, h=$ $1,2, \ldots, L$ and $x_{h i}, h=1,2, \ldots, L, i=1,2, \ldots, n_{h}$.

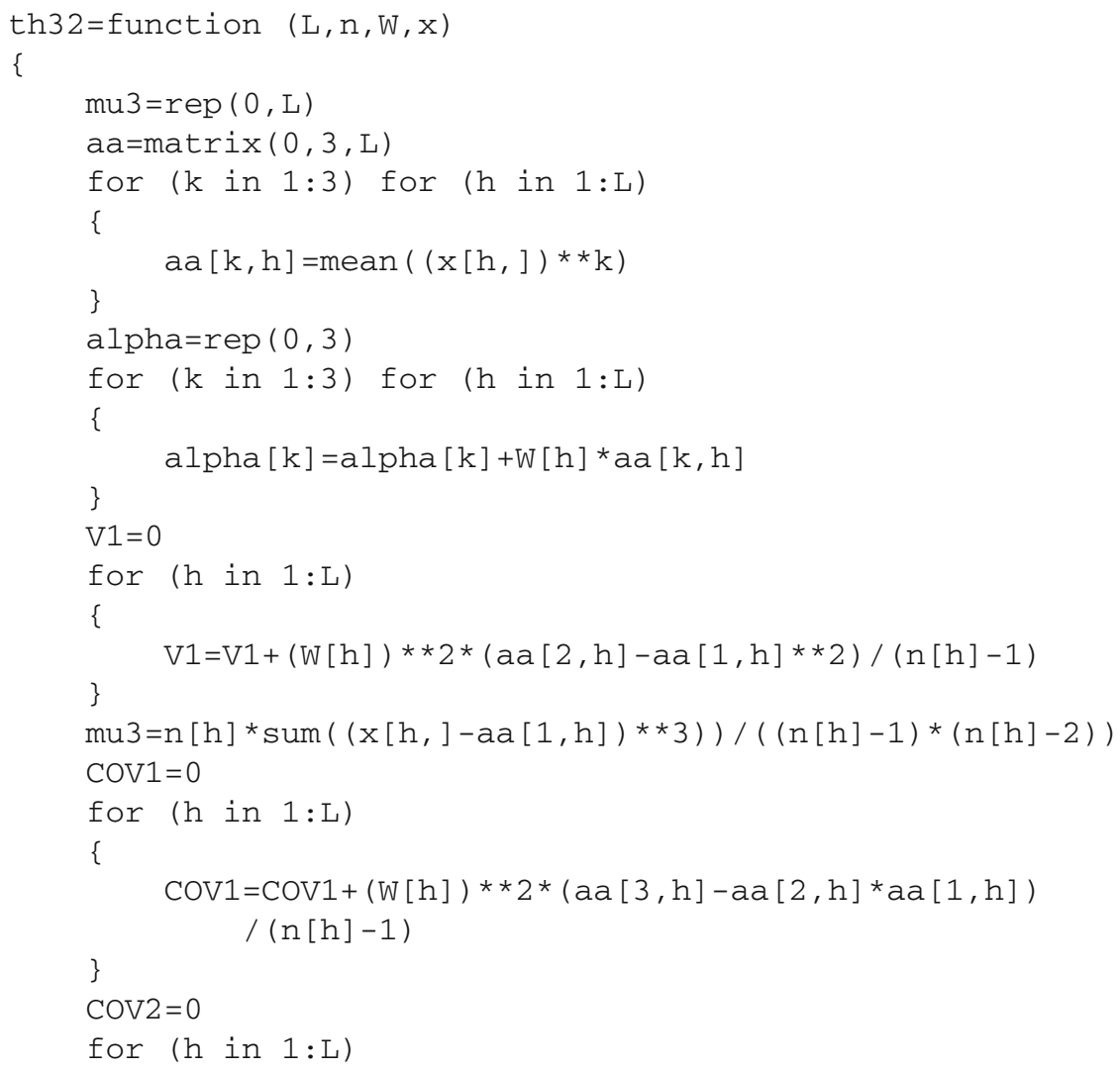




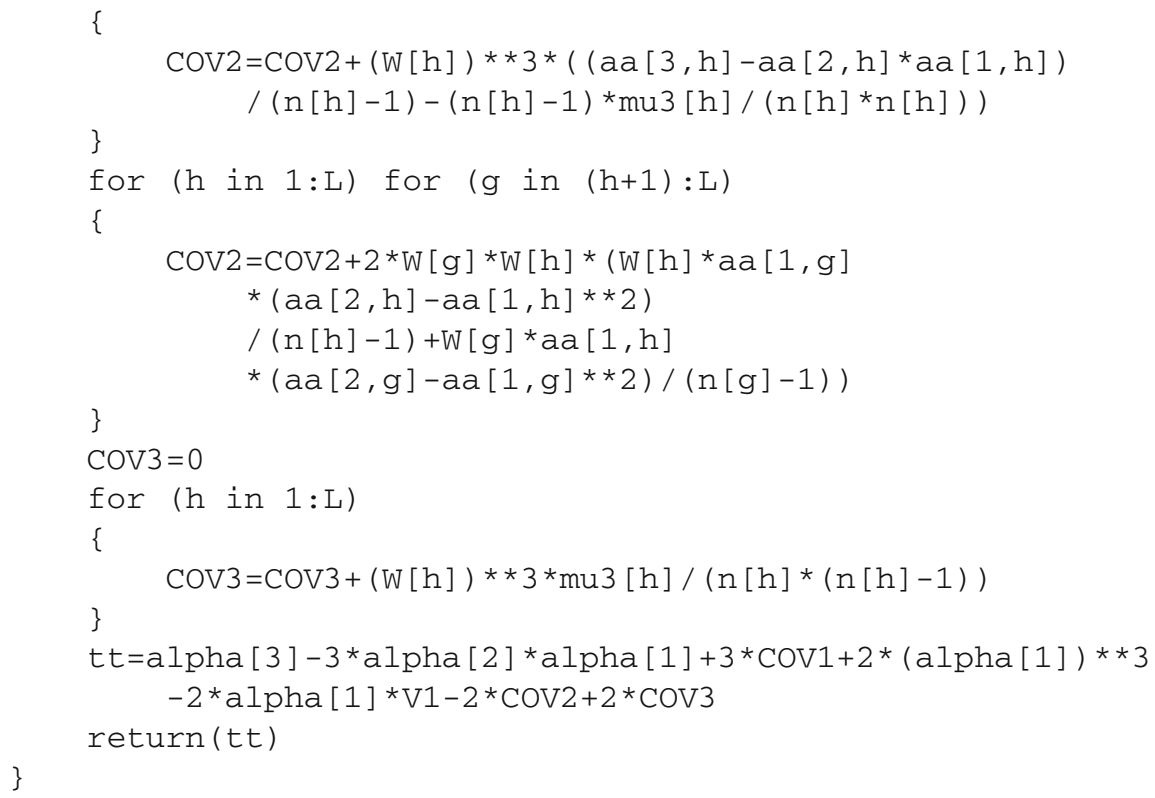

The following function computes the unbiased estimator of the fourth central moment given by Theorem 4.1. The input parameters needed are $n$ and $x_{i}, i=1,2, \ldots, n$.

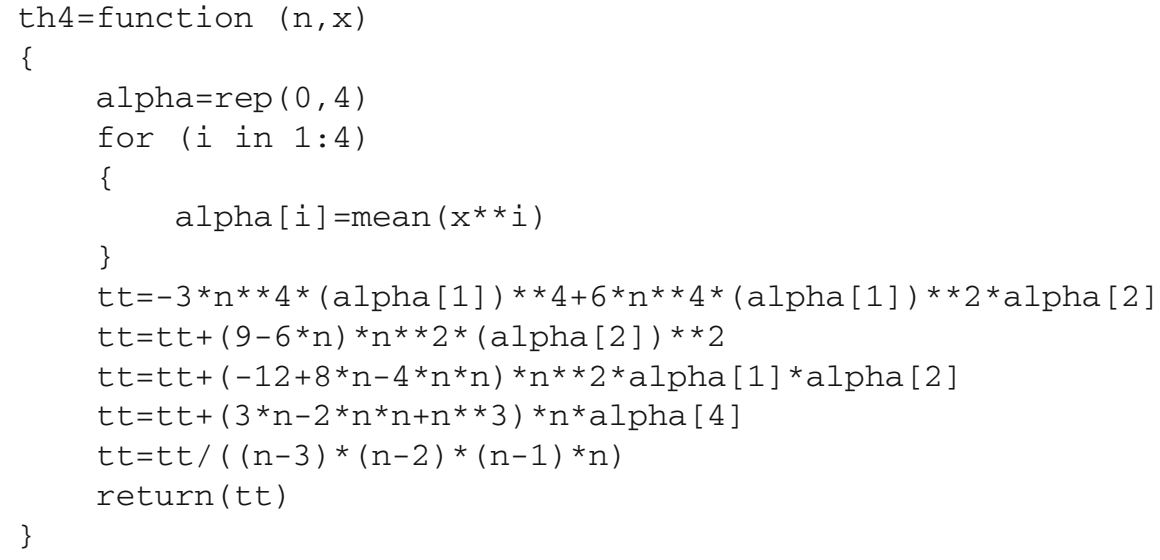

\section{Conclusions and future work}

We have derived several general unbiased estimators for the population central moments of orders two, three and four for all possible statistical populations. Some of these estimators are also valid for other sampling designs broadly used in survey sampling and for sampling theory for finite populations. Moreover, we have proved optimality of several of the derived unbiased estimators under distribution-free setting and for simple random sampling with replacement. Furthermore, we have given simple programs in R for computing all of the derived unbiased estimators. 
The optimal estimators derived in this paper are based on variance. We have used variance because it is the most popular measure for variation and accuracy. However, other measures like skewness and kurtosis could also be used to derive optimal estimators. This is a possible future work.

In this paper, we have considered estimators restricted by unbiasedness. Other restrictions on the class of estimators that one could consider are: mean squared error consistency, consistency based on mean deviations about the mean, consistency based on mean deviations about the median, consistency based on entropy measures, consistency based on the KullbackLeibler divergence measure, and so on.

Results of the kind in Remark 4.4 might be of interest in characterization of the stability of variance estimators under some complex sample designs, provided the specifics related to those designs are explored in additional depth. This is another possible future work.

A final future work is to explore the small-sample/large-sample behavior of the derived results in Sects. 2-4 and to link them with known distributional results.

Acknowledgments The authors would like to thank the Editor-in-Chief and the Associate Editor for careful reading and for their comments which greatly improved the paper.

\section{References}

1. Baillargeon, S., Rivest, L.-P.: Stratification: univariate stratification of survey populations. http://cran. r-project.org/web/packages/stratification/index.html (2012)

2. Barbiero, A., Manzi, G.: SunterSampling: Sunter's sampling design. http://cran.r-project.org/web/ packages/SunterSampling/index.html (2012)

3. Barcaroli, G., Pagliuca, D., Willighagen, E.: SamplingStrata: optimal stratification of sampling frames for multipurpose sampling surveys. http://cran.r-project.org/web/packages/SamplingStrata/index.html (2013)

4. Bellhouse, D.: The central limit theorem under simple random sampling. Stat. Comput. Graph. 55, 352-357 (1997)

5. Blair, G., Imai, K.: List: statistical methods for the item count technique and list experiment. http://cran. r-project.org/web/packages/list/index.html (2013)

6. Brawn, D., Upton, G.: Closed-form parameter estimates for a truncated gamma distribution. Environmetrics 18, 633-645 (2007)

7. Chen, F.: Reweight: adjustment of survey respondent weights. http://cran.r-project.org/web/packages/ reweight/index.html (2012)

8. de Lucia, L.: Stima delle componenti di primo e secondo stadio della convarianza tra le stime delle medie, nel campionamento a due stadi semplici. (Italian) Studi di probabilitá, statistica e ricerca operativa in onore di Giuseppe Pompilj, Edizioni Oderisi, Gubbio, pp. 299-319 (1971)

9. Dodge, Y., Rousson, V.: The complications of the fourth central moment. Am. Stat. 53, 267-269 (1999)

10. D’Orazio, M.: StatMatch: statistical matching. http://cran.r-project.org/web/packages/StatMatch/index. html (2012)

11. Dwyer, P.S.: Moments of any rational integral isobaric sample moment function. Ann. Math. Stat. 8, 21-65 (1937)

12. Erickson, T., Whited, T.M.: Two-step GMM estimation of the errors-in-variables model using high-order moments. Econom. Theory 18, 776-799 (2002)

13. Escobar, E.L., Zamudio, E.B.: SamplingVarEst: sampling variance estimation. http://cran.r-project.org/ web/packages/samplingVarEst/index.html (2012)

14. Gonen, M., Capanu, M.: SvyNom: nomograms for right-censored outcomes from survey designs. http:// cran.r-project.org/web/packages/SvyNom/index.html (2012)

15. Guiso, L., Jappelli, T., Pistaferri, L.: An empirical analysis of earnings and employment risk. J. Bus. Econ. Stat. 20, 241-253 (2002)

16. Gupta, B. C.: Ordered partitions and unbiased estimates of central moments. In: Symmetric Functions in Statistics (Proceedings of theSymposium in Honor of Paul S. Dwyer, University of Windsor, Windsor, Ontario, 1971), University of Windsor, Windsor, Ontario, pp. 131-141 (1972)

17. Gupta, B.C.: Finite moment formulae of $h$-statistics and their estimates. Metrika 22, 85-95 (1975) 
18. Halmos, P.R.: The theory of unbiased estimation. Ann. Math. Stat. 17, 34-43 (1946)

19. Haneuse, S., Saegusa, T., Chaterjee, N., Breslow, N.: osDesign: design and analysis of observational studies. http://cran.r-project.org/web/packages/osDesign/index.html (2012)

20. Hansen, M.H., Hurwitz, W.N.: On the theory of sampling from finite populations. Ann. Math. Stat. 14, 333-362 (1943)

21. Harvey, C.R., Liechty, J.C., Liechty, M.W., Müller, P.: Portfolio selection with higher moments. Quantitative Finance 10, 469-485 (2010)

22. Heffernan, P.M.: Unbiased estimation of central moments by using $U$-statistics. J. R. Stat. Soc. B 59, 861-863 (1997)

23. Herzel, A.: On mean values and unbiased estimators in simple random sampling. Statistica XLII, 315-350 (1982)

24. Ishwaran, H., Takahara, G.: Independent and identically distributed Monte Carlo algorithms for semiparametric linear mixed models. J. Am. Stat. Assoc. 97, 1154-1166 (2002)

25. JacqminGadda, H., Commenges, D.: Tests of homogeneity for generalized linear models. J. Am. Stat. Assoc. 90, 1237-1246 (1995)

26. Kadilar, C., Unyazici, Y., Cingi, H.: Ratio estimator for the population mean using ranked set sampling. Stat. Pap. 50, 301-309 (2009)

27. Kankainen, A., Taskinen, S., Oja, H.: Tests of multinormality based on location vectors and scatter matrices. Stat. Methods Appl. 16, 357-379 (2007)

28. Kincaid, T., Olsen, T.: spsurvey: spatial survey design and analysis. http://cran.r-project.org/web/ packages/spsurvey/index.html (2012)

29. Li, Z.X.: Estimation in linear mixed models for longitudinal data under linear restricted conditions. J. Stat. Plan. Inference 141, 869-876 (2011)

30. Lopez-Valcarce, R., Mosquera, C.: Sixth-order statistics-based non-data-aided SNR estimation. IEEE Commun. Lett. 11, 351-353 (2007)

31. Lumley, T.: Survey: analysis of complex survey samples. http://cran.r-project.org/web/packages/survey/ index.html (2012)

32. Manitz, J.: Samplingbook: survey sampling procedures. http://cran.r-project.org/web/packages/ samplingbook/index.html (2013)

33. Maritz, J.S.: Distribution-Free Statistical Methods, 2nd edn. Chapman and Hall, London (1995)

34. Mikhail, N.N., Malik, H.J.: Unbiased estimates of Dwyer's moment function and their products for finite population. J. Indian Stat. Assoc. 16, 71-82 (1978)

35. Montanari, A., Viroli, C.: A skew-normal factor model for the analysis of student satisfaction towards university courses. J. Appl. Stat. 37, 473-487 (2010)

36. Neely, W.W.: RDS: R functions for respondent-driven sampling. http://cran.r-project.org/web/packages/ RDS/index.html (2009)

37. Oberski, D.: lavaan.survey: complex survey structural equation modeling. http://cran.r-project.org/web/ packages/lavaan.survey/index.html (2013)

38. Olkin, I.: Multivariate ratio estimation for finite populations. Biometrika 45, 154-165 (1958)

39. Polo, C.: On the sample variance. XIV Congreso Nacional de Estadística, Investigación Operativa e Informática. Tomo I, Caja General de Ahorros, Granada, pp. 393-405 (1984)

40. R Development Core Team: R: a Language and Environment for Statistical Computing: R Foundation for Statistical Computing. Austria, Vienna (2013)

41. Raj, D.: Sampling Theory. McGraw-Hill, New York (1968)

42. Rao, J.N.K.: On the estimation of the relative efficiency of sampling procedures. Ann. Inst. Stat. Math. 14, 143-150 (1962)

43. Rojas, H.A.G.: TeachingSampling: sampling designs and parameter estimation in finite population. http:// cran.r-project.org/web/packages/TeachingSampling/index.html (2012)

44. Rose, C., Smith, M.D.: h-statistics: unbiased estimators of central moments, pp. 253-256. Mathematical Statistics with Mathematica. Springer, New York (2002)

45. Espejo, Ruiz: M., Delgado Pineda, M.: On variance estimation for post stratification: a review. Metron 55, 209-220 (1997)

46. Ruiz Espejo, M., Ruiz Espejo, M.M.: Equilibrated strategy for population variance estimation. Test 1, 79-91 (1992)

47. Sánchez-Crespo, J.L.: A new sampling scheme: selection with graduated variable probabilities without replacement. Bull. Int. Stat. Inst. 47, 458-461 (1977)

48. Shimizu, S., Kana, Y.: Use of non-normality in structural equation modeling: application to direction of causation. J. Stat. Plan. Inference 138, 3483-3491 (2008)

49. Shiraito, Y., Imai, K.: Endorse: R package for analyzing endorsement experiments. http://cran.r-project. org/web/packages/endorse/index.html (2013) 
50. Silva, I., Silva, M.E.: Parameter estimation for INAR processes based on high-order statistics. Revstat 7, 105-117 (2009)

51. Singh, H.P., Tailor, R., Singh, S., Kim, J.M.: A modified estimator of population mean using power transformation. Stat. Pap. 49, 37-58 (2008)

52. Singh, S.: Generalized calibration approach for estimating variance in survey sampling. Ann. Inst. Stat. Math. 53, 404-417 (2001)

53. Soliman, S.S., Hsue, S.Z.: Signal classification using statistical moments. IEEE Trans. Commun. 40, 908-916 (1992)

54. Stafford, J.E., Bellhouse, D.R.: A computer algebra for sample survey theory, pp. 19-29. In: Proceedings of the Survey Methods Section, Statistical Society of Canada (1996)

55. Stafford, J.E., Bellhouse, D.R.: A computer algebra for sample survey theory. Surv. Methodol. 23, 3-10 (1997)

56. Stuart, A., Ord, K.: Kendall's Advanced Theory of Statistics, vol. I: Distribution Theory, 6th edn. Edward Arnold, London (1994)

57. Tillé, Y., Matei, A.: Sampling: survey sampling. http://cran.r-project.org/web/packages/sampling/index. html (2012)

58. Tucker, H.G.: Mathematical Methods in Sample Surveys. World Scientific, Singapore (1998)

59. van de Pol, M.: odprism: optimal design and performance of random intercept and slope models. http:// cran.r-project.org/web/packages/odprism/index.html (2011)

60. Verbeke, T.: SDaA: sampling: design and analysis. http://cran.fhcrc.org/web/packages/SDaA/index.html (2011)

61. Wolfram Research Inc.: Mathematica, Version 8.0, Champaign, Illinois (2010)

62. Wu, J.H., Su, W.H.: Estimation of moments for linear panel data models with potential existence of time effects. Stat. Probab. Lett. 80, 1933-1939 (2010)

63. Wu, P., Zhu, L.X.: An orthogonality-based estimation of moments for linear mixed models. Scand. J. Stat. 37, 253-263 (2010)

64. Yuan, K.H., Jennrich, R.I.: Estimating equations with nuisance parameters: theory and applications. Ann. Inst. Stat. Math. 52, 343-350 (2000)

65. Zacks, S.: The Theory of Statistical Inference. Wiley, New York (1971) 